\title{
Notes: Is Abraham Damping Constant and Inertia in Matter A Consequent Echo of Its Future State?
}

\author{
Anthony Maccini \\ Flat 9, Fairhall Court, 114 - 124 Kingcharles Road, Surbiton, KT5 8QL, England \\ *Corresponding author details: Anthony Maccini; anthonymaccini@hotmail.com
}

\begin{abstract}
I show in this paper that the Wheeler-Feynman Absorber theory is based on advanced and retarded waves in matter, known as electrical inertia or known as the Abraham damping constant, where inertia is regarded as an echo from matter's future state, in its resistance to acceleration, then I show through calculations on the Abraham damping constant that others have done, leads to advanced waves being responsible for this damping constant, lending support to the Wheeler-Feynman Absorber theory and the work of Darko Bajlo, who first detected advanced waves in his experiments on 3rd December 2016 to 5th January 2017, and that such experiments were modelled on the Wheeler-Feynman Absorber theory and lends more support for this.
\end{abstract}

Keywords: advanced waves; Abraham damping constant; electrical inertia; Wheeler-Feynman Absorber theory

\section{INTRODUCTION}

In the Wheeler-Feynman Absorber theory [1], particles that get excited send out advanced and retarded waves to other particles exciting them, to also send out advanced and retarded waves that go back to their source and creates what is known as electrical inertia, leading to the Abraham damping constant, of which many of the paradoxes of quantum mechanics can be explained on this basis. Bajlo [2] first detected advanced waves, whose experiment was based on the model of the WheelerFeynman Absorber theory and confirms this; that looking in detail at the Abraham Lorentz force and its calculations, leads to advanced signals from the future which supports the Wheeler-Feynman Absorber theory.

\section{WHEELER-FEYNMAN ABSORBER THEORY}

According to the Wheeler-Feynman Absorber theory, when a charged particle jiggles it radiates both into the past and into the future. This causes another charged particle to jiggle in the future. The jiggling of that particle sends another wave both into the future and into the past. The two sets of waves cancel out everywhere, except in the region between the two charged particles. But because one wave goes forward in time and the other travels backward in time, the connection is made instantaneously and radiation resistance or inertia of the mass results. This effect instantaneously produces a kind of electrical inertia. This can be incorporated into a model of gravity and inertia, that operates at the quantum level.

There is another interpretation that adopts the WheelerFeynman Absorber theory, known as the Transactional interpretation [3] where advanced effects are described as a natural process going on in nature. From this, it seems that many of the paradoxes of the quantum mechanics can be explained with the notion of advanced effects. Feynman suggested in his Book [4] QED the strange theory of light and matter, page 98, 'This phenomenon is general. Every particle in nature has an amplitude to move backwards in time, and therefore has an anti-particle'.

Feynman did a calculation on the field in relation to the Wheeler-Feynman Absorber theory:

$$
\begin{aligned}
\operatorname{Etot}(x, t)= & \sum_{n} \frac{\operatorname{Eret} n(x, t)+\operatorname{Eadv} n(x, t)}{2} \\
\operatorname{Efree}(x, t)= & \sum_{n} \frac{\operatorname{Eret} n(x, t)-\operatorname{Eadv} n(x, t)}{2}=0 \\
\operatorname{Etot}(x, t)= & \sum_{n} \frac{\operatorname{Eret} n(x, t)+\operatorname{Eadv} n(x, t)}{2} \\
& +\sum_{n} \frac{\operatorname{Eret} n(x, t)-\operatorname{Eadv} n(x, t)}{2} \\
= & \sum_{n} \operatorname{Eret} n(x, t)
\end{aligned}
$$

Where Etot is the total field and where Eret and Eadv is the retarded and advanced field.

The resulting wave appears to have a preferred time direction, because it respects causality. However, this is only an illusion, indeed it is always possible to reverse the time direction by simply exchanging emitter and absorber:

$$
\sum_{n} \operatorname{Eadv} n(x, t)
$$

Indeed Bajlo who first detected advanced waves in his experiment (3rd December 2016 to 5th January 2017) based his experiment on the Wheeler-Feynman Absorber theory and got positive results, as he says in his paper [2] 'For the purpose of detecting advanced electromagnetic radiation predicted by Wheeler-Feynman absorber theory for the case of incomplete absorption of retarded electromagnetic radiation, pulses in duration of $6 \mathrm{~ns}$ to 24 $\mathrm{ns}$, wavelength from $91 \mathrm{~cm}$ to $200 \mathrm{~cm}$ where supplied to three different transmitting antennas. Detection was done with a monopole antenna in the advanced time window at a time $2 \mathrm{r} / \mathrm{c}$ before the arrival of the centre of the retarded pulse. 
At distances ranging from $230 \mathrm{~cm}$ to $18 \mathrm{~m}$, advanced signals were measured in the range from 15.4 to $30.9^{\prime}$. Indeed, this is experimental evidence confirming the Wheeler-Feynman Absorber theory. But does not the fact of the Wheeler-Feynman Absorber theory of advanced fields imply that the future and past exist as realities, even more so with Bajlo, with his experiment in discovering for the first time advanced waves that are travelling into the past, and Bajlo used an absorber in his experiment based on the Wheeler-Feynman Absorber theory and got positive results of advanced signals, before the retarded waves were emitted.

As Bajlo says [5] in his paper, 'In the act of observation lies a mechanism that masks advanced electromagnetic waves. The advanced waves of the receiving antenna cancel out the advanced waves of the transmitting antenna in the direction of the receiving antenna. In other direction in which there are no absorbers, the transmitting antenna radiates advanced electromagnetic waves into free space. The act of observation itself creates an illusion that the electromagnetic arrow points only in the direction of the future. Is there any way to detect advanced electromagnetic waves that the transmitting antenna radiates into free space. The solution to this measurement problem is to minimize the influence of the measuring instrument on the phenomenon being measured. This can be easily achieved with the meter and decimeter radio waves by using a small enough receiving antenna for detection, where its advanced waves cannot completely cancel advanced waves from transmitting antenna. If the results of the recent experiments represent the real signal and not some systematic error, then the advanced radiation can actually be detected with a receiving antenna twenty times smaller than the wavelength of emitted electromagnetic pulses.

There is another interesting effect that is explained from the Wheeler-Feynman Absorber theory, where we have radiation resistance and Absorber theory of radiation. But this leads to the effect of inertia or the damping constant $\mathrm{T}$. Electrical inertia, and this simply means that gravity and inertia are the result of advanced energy in matter, of matter responding to its own echo of its own future state. The result of advanced effects in matter causes the Abraham-Lorentz damping constant:

$$
T=2 e^{2} / 3 m^{\circ} c^{\wedge} 2
$$

Where $T$ is the Abraham-Lorentz damping constant. That this mechanism of electrical inertia of advanced effects of radiation:

$$
\sum_{n} \operatorname{Eadv} n(x, t)
$$

This is the cause of the $T$ Abraham-Lorentz damping constant.

\section{ABRAHAM - LORENTZ FORCE}

I looked into the Abraham-Lorentz force $T$, which is the result of the most fundamental calculation of the effect of self-generated fields. General relativity has an unsolved self-field problem. And at the present there are problems of calculating self-generated fields of single particles, problems of infinities in their calculations. This is an unsolved self-field problem. Also, with the AbrahamLorentz force $T$, there is the result they get in their calculations of $T$, of signals from the future, and this is a problem for them. For a particle in an external force Fext, we have:

$$
m v=F r a d+F e x t=m t^{\circ} v+F e x t
$$

where, $t^{\circ}=\frac{\mathrm{U}^{\circ} q^{\wedge} 2}{6 \pi m c}$

Where $U^{\circ}$ is Permeability of free space.

This equation can be integrated once to obtain:

$$
m v=\frac{1}{t^{\circ}} \int_{t}^{\infty} \operatorname{ext}\left(-\frac{t^{\prime}-t}{t^{\circ}}\right) F \operatorname{ext}\left(t^{\prime}\right) d t^{\prime}
$$

The integral extends from the present to infinitely far in the future. Thus, future values of the force affect the acceleration of the particle in the present. The future values are weighted by the factor:

\section{$\left(-\frac{t^{\prime}-t}{t^{\circ}}\right)$ Which falls off rapidly for times greater than $t^{\circ}$}

in the future. Therefore, signals from an interval approximately $t^{\circ}$, into the future affect the acceleration in the present. For an electron it is $10^{\wedge} 24 \mathrm{sec}$. With Lienards relativistic generalization of Larmar formula in the comoving frame, one can show this to be a valid force by manipulating the time average equation for power:

$$
P=\frac{\cup^{\circ} q^{\wedge} 2 a^{\wedge} 2 y^{\wedge} 2}{6 \pi c}
$$

Where $\mathrm{P}=$ power and $\mathrm{U}^{\circ}$, is permeability of free space.

There are solutions using the Abraham-Lorentz-Dirac equation that anticipates a change in the external force and according to which the particle accelerates in advance of the application of a force! The so called pre-acceleration solutions:

$$
\frac{1}{\Delta t} \int_{0}^{t} P d t=\frac{1}{\Delta t} \int_{0}^{t} F \cdot V d t
$$

So, what I found out about the damping constant $T$, in physics is full of these problems they have with advanced effects in their calculations of it, and it lends support to Wheeler-Feynman Absorber theory of being the cause of $T$, inertia.

\section{CONCLUSION}

It appears that there is support for the Wheeler-Feynman Absorber theory from Bajlo's experiment in detecting advanced waves and the calculations of Abraham Lorentz force, which are all based on advanced waves getting back to their source and whose] natural result is electrical inertia or the Abraham damping constant, which suggests that one has advanced effects in matter commonly known as inertia, which must be considered as an echo from the future state of that matter itself, being its resistance to acceleration.

\section{REFERENCES}

[1] John A. Wheeler, and Richard P. Feynman. Interaction with the Absorber as the mechanism of radiation. Reviews of modern physics, Volume 17, numbers 2 and 3, April-July, (1945) pp157-181

[2] Bajlo D. Measurement of advanced electromagnetic radiation, Zenodo doi: 10,5281/zenodo. 247283. (2017)

[3] John Carmer. The Quantum handshake explored, Journal of the British interplanetary society, Vol. 70 (2017) pp.372-384

[4] Richard P. Feynman, QED the strange theory of light and matter, published in Great Britain by Penguin Books (1990) p98

[5] Bajlo D. The hidden arrow of electromagnetic radiation: unmasking advanced waves. (September 2017) 